\title{
State and Parameter Estimation for Nonlinear Systems: a Takagi-Sugeno Approach
}

\author{
Souad Bezzaoucha, Benoît Marx, Didier Maquin, José Ragot
}

\begin{abstract}
The main contribution of this paper is to propose a systematic approach to observer design for nonlinear TakagiSugeno (T-S) time-varying systems. The proposed procedure is based on the sector nonlinearity approach using the convex polytopic transformation. The exact writing of the time-varying nonlinear system as a T-S model allows to provide a state and parameter estimation.

Index Terms-Time-varying nonlinear systems, TakagiSugeno models, sector nonlinearity approach, convex polytopic transformation, state and parameter observer, linear matrix inequality.
\end{abstract}

\section{INTRODUCTION}

The observer design for nonlinear systems can be viewed as the heart of system control and model-based diagnosis [5], [2]. Unfortunately, the introduction of time-varying parameters in the system models, needed to accurately represent the system behaviour, leads to more challenging problems in estimation.

In the present work, a focus is made on the time-varying parameter systems, where the parameter variations are inaccessible (non measurable) and may be used as model faults (acting as disturbances or/and uncertainties). In this case, a conventional observer cannot be used directly, and so called adaptive observers developed for joint state and unknown parameter estimation have to be implemented.

Numerous approaches were proposed in order to deal with observer design for nonlinear systems [14], [11], [3], [6]. An efficient way consists in rewriting the original nonlinear system in a simplier form, like the T-S one. Originaly introduced by [12], the T-S representation allows to describe the exact nonlinear behaviour of a system, under the condition that its nonlinearities are bounded. This is reasonable as variables of physical systems are real and always bounded. See for example [1], [8] and the references therein.

The T-S formalism is based on a time-varying interpolation between linear submodels. The global model is a convex combination of the $r$ submodels [13]:

$$
\left\{\begin{array}{l}
\dot{x}(t)=\sum_{i=1}^{r} \mu_{i}(\xi(t))\left(A_{i} x(t)+B_{i} u(t)\right) \\
y(t)=\sum_{i=1}^{r} \mu_{i}(\xi(t))\left(C_{i} x(t)+D_{i} u(t)\right)
\end{array}\right.
$$

where $x(t) \in \mathbb{R}^{n_{x}}$ is the system state variable, $u(t) \in \mathbb{R}^{n_{u}}$ is the control input and $y(t) \in \mathbb{R}^{m}$ is the system output. $\xi(t) \in \mathbb{R}^{q}$ is the decision variable vector assumed to be either

The authors are with Université de Lorraine, CRAN, UMR 7039, 2 avenue de la Forêt de Haye, Vandoeuvre-lès-Nancy Cedex, 54516, France. CNRS, CRAN, UMR 7039, France. firstname.name@univ-lorraine.fr measurable (as the system output), known (as the system input) or unmeasurable (as the system state). The weighting functions $\mu_{i}(\xi(t))$ of the $r$ submodels satisfy the convex sum property

$$
\left\{\begin{array}{l}
\sum_{i=1}^{r} \mu_{i}(\xi(t))=1 \\
0 \leq \mu_{i}(\xi(t)) \leq 1, \quad i=1, \ldots, r
\end{array}\right.
$$

This representation is very interesting in the sense that it simplifies the mathematical manipulation compared to the original nonlinear models. It allows to extend the use of some tools developed in the linear framework to the nonlinear systems, for the stability study, the control design and observer synthesis.

A systematic procedure to transform a nonlinear system by rewriting it into a T-S form, without any loss of information is known as the sector nonlinearity transformation (SNT) [13], [9]. This transformation advantage is to be analytical and systematic. Based on the nonlinear equations of the original model, a quasi-Linear Parameter Varying (quasiLPV) is written. The local models of this form are state and control input affine, however, this representation is not unique. Depending on the objectives, many quasi-LPV forms may be obtained [9].

Using the convex polytopic transformation, a T-S model is obtained with all the nonlinearities being transfered into the weighting functions. Although the polytopic transformation leads to T-S models with unmeasurable premisse variables, most of the works on T-S systems are devoted to T-S models with known premisse variables, since they are obviously easier to study.

The present work deals with nonlinear time-varying parameter systems described by T-S models with unmeasurable premise variables. The main difficuly here is the joint state and parameter estimation, since the premise variables are unmeasurable and depend on the state that need to be estimated.

The proposed methods consists in rewriting the varying parameters and the nonlinearities in a polytopic form allowing to transform the original nonlinear system into T-S model. The T-S model obtained with the sector nonlinearity transformation has the major interest to be analytically equivalent to the original time-varying nonlinear system.

Up to the author's knowledge, this is the first contribution where the time-varying parameter and state estimation problem is addressed in such a way. This allows to design a state and parameter observer by minimizing the $\mathscr{L}_{2}$-gain from the parameter to the state and parameter estimation errors. Using 
the Lyapunov theory, the $\mathscr{L}_{2}$ gain minimization is expressed as a minimization problem under LMI constraints.

The paper is organized as follows. Section II introduces the T-S representation of the nonlinear time-varying parameter system. In section III, joint state and unknowm time-varying parameter estimation is proposed for T-S systems with unmeasurable premise variables. An application of the proposed approach to an activated sludge reactor model with some simulation results are given in section IV. Conclusions are detailed in section $\mathrm{V}$.

\section{Problem Statement: T-S Modelling of NONLINEAR TIME-VARYING PARAMETER}

A first contribution of this work is to model nonlinear time-varying systems using the T-S representation. For that, each time-varying parameter is rewritten under a particular form.

Let us consider the nonlinear time-varying T-S system represented by equation (3) with $n$ parameters $\theta_{j}(t)$

$$
\left\{\begin{aligned}
\dot{x}(t) & =\sum_{i=1}^{r} \mu_{i}(x(t))\left(A_{i}(t) x(t)+B_{i}(t) u(t)\right) \\
y(t) & =C x(t)
\end{aligned}\right.
$$

with

$$
\left\{\begin{array}{l}
A_{i}(t)=A_{i}+\sum_{j=1}^{n} \theta_{j}(t) \bar{A}_{i j} \\
B_{i}(t)=B_{i}+\sum_{j=1}^{n} \theta_{j}(t) \bar{B}_{i j}
\end{array}\right.
$$

Remark 1: In (4), it is supposed that the matrices $A_{i}(t)$ and $B_{i}(t)$ depend on the same parameters. However, if a parameter $\theta_{j}(t)$ does not affect $A_{i}(t)$ (resp. $B_{i}(t)$ ), then the corresponding matrix $\bar{A}_{i j}$ (resp. $\bar{B}_{i j}$ ) is null.

$A_{i}, \bar{A}_{i j}, B_{i}, \bar{B}_{i j}$ are known matrices with suitable dimensions. $\theta_{j}(t)$ are time-varying parameters. They are nonmeasurable but bounded $\theta_{j}(t) \in\left[\theta_{j}^{2}, \theta_{j}^{1}\right]$. Each parameter $\theta_{j}(t)$ is expressed as:

$$
\theta_{j}(t)=\widetilde{\mu}_{j}^{1}\left(\theta_{j}(t)\right) \theta_{j}^{1}+\widetilde{\mu}_{j}^{2}\left(\theta_{j}(t)\right) \theta_{j}^{2}
$$

with

$$
\begin{aligned}
& \left\{\begin{aligned}
\widetilde{\mu}_{j}^{1}\left(\theta_{j}(t)\right) & =\frac{\theta_{j}(t)-\theta_{j}^{2}}{\theta_{j}^{1}-\theta_{j}^{2}} \\
\widetilde{\mu}_{j}^{2}\left(\theta_{j}(t)\right) & =\frac{\theta_{j}^{1}-\theta_{j}(t)}{\theta_{j}^{1}-\theta_{j}^{2}}
\end{aligned}\right. \\
& \widetilde{\mu}_{j}^{1}\left(\theta_{j}(t)\right)+\widetilde{\mu}_{j}^{2}\left(\theta_{j}(t)\right)=1, \forall t
\end{aligned}
$$

Replacing (5) in (4), it becomes:

$$
\begin{aligned}
& A_{i}(t)=A_{i}+\sum_{j=1}^{n} \sum_{k=1}^{2} \widetilde{\mu}_{j}^{k}\left(\theta_{j}(t)\right) \theta_{j}^{k} \bar{A}_{i j} \\
& B_{i}(t)=B_{i}+\sum_{j=1}^{n} \sum_{k=1}^{2} \widetilde{\mu}_{j}^{k}\left(\theta_{j}(t)\right) \theta_{j}^{k} \bar{B}_{i j}
\end{aligned}
$$

In order to have the same weighting functions for all the time-varying matrices $A_{i}(t)$ and $B_{i}(t)$, exploiting the convex sum property of the weighting functions $\widetilde{\mu}_{j}^{1}(t)$ and $\widetilde{\mu}_{j}^{2}(t)$ of each parameter $\theta_{j}(t),(7)$ is written as:

$$
\left\{\begin{aligned}
A_{i}(t)= & A_{i}+\sum_{j=1}^{n}\left[\left[\left(\widetilde{\mu}_{j}^{1}\left(\theta_{j}(t)\right) \theta_{j}^{1}+\widetilde{\mu}_{j}^{2}\left(\theta_{j}(t)\right) \theta_{j}^{2}\right) \bar{A}_{i j}\right]\right] \times \\
& {\left[\prod_{\substack{k=1 \\
k \neq j}}^{n} \sum_{m=1}^{2} \widetilde{\mu}_{k}^{m}\left(\theta_{k}(t)\right)\right] } \\
= & A_{i}+\sum_{j=1}^{2^{n}} \widetilde{\mu}_{j}(t) \overline{\mathscr{A}}_{i j} \\
B_{i}(t)= & B_{i}+\sum_{j=1}^{n}\left[\left[\left(\widetilde{\mu}_{j}^{1}\left(\theta_{j}(t)\right) \theta_{j}^{1}+\widetilde{\mu}_{j}^{2}\left(\theta_{j}(t)\right) \theta_{j}^{2}\right) \bar{B}_{i j}\right]\right] \times \\
& {\left[\prod_{\substack{k=1 \\
k \neq j}}^{n} \sum_{m=1}^{2} \widetilde{\mu}_{k}^{m}\left(\theta_{k}(t)\right)\right] } \\
= & B_{i}+\sum_{j=1}^{2^{n}} \widetilde{\mu}_{j}(t) \overline{\mathscr{B}}_{i j}
\end{aligned}\right.
$$

with

$$
\left\{\begin{array}{l}
\widetilde{\mu}_{j}(\theta(t))=\prod_{k=1}^{n} \widetilde{\mu}_{k}^{\sigma_{j}^{k}}\left(\theta_{k}(t)\right) \\
\overline{\mathscr{A}}_{i j}=\sum_{k=1}^{n} \theta_{k}^{\sigma_{j}^{k}} \bar{A}_{i k} \\
\overline{\mathscr{B}}_{i j}=\sum_{k=1}^{n} \theta_{k}^{\sigma_{j}^{k}} \bar{B}_{i k}
\end{array}\right.
$$

The indices $\sigma_{j}^{k}$ equal to 1 or 2 , indicate which partition of the $k^{t h}$ parameter $\left({\widetilde{\mu_{k}}}^{1}\right.$ or ${\widetilde{\mu_{k}}}^{2})$ is involved in the $j^{t h}$ submodel. The relation between the submodel number $j$ and the $\sigma_{j}^{k}$ indices are given by the following equation

$$
j=2^{n-1} \sigma_{j}^{1}+2^{n-2} \sigma_{j}^{2}+\ldots+2^{0} \sigma_{j}^{n}-\left(2^{1}+2^{2}+\ldots+2^{n-1}\right)
$$

Equation (8) presents the advantage to have the same global convex weighting functions for $A_{i}(t)$ and $B_{i}(t)$ (see [4] for calculation details).

In the following, $\theta(t)$ is the vector of components $\theta_{j}(t), j=$ $1, \ldots, n$.

Finally, using equations (8) and (4), (3) becomes:

$$
\begin{gathered}
\dot{x}(t)=\sum_{i=1}^{r} \sum_{j=1}^{2^{n}} \mu_{i}(x(t)) \widetilde{\mu}_{j}(\theta(t))\left(\mathscr{A}_{i j} x(t)+\mathscr{B}_{i j} u(t)\right) \\
y(t)=C x(t) \\
\mathscr{A}_{i j}=A_{i}+\overline{\mathscr{A}}_{i j} \\
\mathscr{B}_{i j}=B_{i}+\overline{\mathscr{B}}_{i j}
\end{gathered}
$$

\section{STATE AND TIME-VARYING PARAMETER OBSERVER}

Based on the obtained T-S model, a simultaneous state and parameter observer may be designed and implemented. An $\mathscr{L}_{2}$ attenuation approach will be proposed to minimize the effect of the time-varying parameters on the state and parameter error estimation. 
The state and parameter observer is taken as the following

$$
\left\{\begin{array}{l}
\dot{\hat{x}}(t)=\sum_{i=1}^{r} \sum_{j=1}^{2^{n}} \mu_{i}(\hat{x}(t)) \widetilde{\mu}_{j}(\hat{\theta}(t)) \\
\dot{\hat{\theta}}(t)=\sum_{i=1}^{r} \sum_{j=1}^{2^{n}} \mu_{i j}\left(\hat{x}(t)+\mathscr{B}_{i j} u(t)+L_{i j}(\hat{y}(t)-y(t))\right) \\
\left(K_{i j}(y(t)-\hat{\theta}(t))\right. \\
\hat{y}(t)=C \hat{x}(t) \quad
\end{array}\right.
$$

where $L_{i j} \in \mathbb{R}^{n_{x} \times m}, K_{i j} \in \mathbb{R}^{n \times m}$ and $\alpha_{i j} \in \mathbb{R}^{n \times n}$ are the gains to be determined such that the estimated state and parameter converge to the system state and parameter.

Let us define the state estimation error $e_{x}(t)$ as

$$
e_{x}(t)=x(t)-\hat{x}(t)
$$

Its dynamics cannot be easily computed directly from (14) since in equation (11) the weighting functions of the state depends on the unmeasurable variables $(\theta(t)$ and $x(t))$. Because of that, based on the convex sum property of the weighting functions, the state equation (11) is rewritten as follow

$$
\begin{gathered}
\dot{x}(t)=\sum_{i=1}^{r} \sum_{j=1}^{2^{n}}\left[\mu_{i}(\hat{x}(t)) \widetilde{\mu}_{j}(\hat{\theta}(t))\left(\mathscr{A}_{i j} x(t)+\mathscr{B}_{i j} u(t)\right)+\right. \\
\left.\left(\mu_{i}(x(t)) \widetilde{\mu}_{j}(\theta(t))-\mu_{i}(\hat{x}(t)) \widetilde{\mu}_{j}(\hat{\theta}(t))\right)\left(\mathscr{A}_{i j} x(t)+\mathscr{B}_{i j} u(t)\right)\right]
\end{gathered}
$$

This form allows a better comparison of $x(t)$ with $\hat{x}(t)$, since $\mu_{i}(\hat{x}(t)) \widetilde{\mu}_{j}(\hat{\theta}(t))$ not only appears in (13), but also in (15). Let us define:

$$
\begin{aligned}
\Delta A(t) & =\sum_{i=1}^{r} \sum_{j=1}^{2^{n}}\left[\mu_{i}(x(t)) \widetilde{\mu}_{j}(\theta(t))-\mu_{i}(\hat{x}(t)) \widetilde{\mu}_{j}(\hat{\theta}(t))\right] \mathscr{A}_{i j} \\
& =\mathscr{A} \Sigma_{A}(t) E_{A}
\end{aligned}
$$

and

$$
\begin{aligned}
\Delta B(t) & =\sum_{i=1}^{r} \sum_{j=1}^{2^{n}}\left[\mu_{i}(x(t)) \widetilde{\mu}_{j}(\theta(t))-\mu_{i}(\hat{x}(t)) \widetilde{\mu}_{j}(\hat{\theta}(t))\right] \mathscr{B}_{i j} \\
& =\mathscr{B} \Sigma_{B}(t) E_{B}
\end{aligned}
$$

with

$$
\begin{aligned}
& \mathscr{A}=\left[\begin{array}{lll}
\mathscr{A}_{11} & \ldots & \mathscr{A}_{r 2^{n}}
\end{array}\right], \Sigma_{A}(t)=\operatorname{diag}\left(\delta_{11}(t), \ldots, \delta_{r 2^{n}}(t)\right), \\
& \mathscr{B}=\left[\begin{array}{lll}
\mathscr{B}_{11} & \ldots & \mathscr{B}_{r 2^{n}}
\end{array}\right], \Sigma_{B}(t)=\operatorname{diag}\left(\delta_{11}(t), \ldots, \delta_{r 2^{n}}(t)\right), \\
& E_{A}=\left[\begin{array}{lll}
I_{n_{x}} & \ldots & I_{n_{x}}
\end{array}\right]^{T}, E_{B}=\left[\begin{array}{lll}
I_{n_{u}} & \ldots & I_{n_{u}}
\end{array}\right]^{T} \\
& \delta_{i j}(t)=\mu_{i}(x(t)) \widetilde{\mu}_{j}(\theta(t))-\mu_{i}(\hat{x}(t)) \widetilde{\mu}_{j}(\hat{\theta}(t))
\end{aligned}
$$

where $\operatorname{diag}\left(A_{1}, \ldots, A_{n}\right)$ refers to a block diagonal matrix with the square matrices $A_{1}, \ldots, A_{n}$ on its diagonal.

Thanks to property (2), we also have

$$
-1 \leq \delta_{i j}(t) \leq 1
$$

which implies from definition (18)

$$
\Sigma_{A}^{T}(t) \Sigma_{A}(t) \leq I, \quad \Sigma_{B}^{T}(t) \Sigma_{B}(t) \leq I
$$

Using (16) and (17), the system (15) is then written as an uncertain system given by:

$$
\begin{aligned}
\dot{x}(t)=\sum_{i=1}^{r} \sum_{j=1}^{2^{n}} \mu_{i}(\hat{x}(t)) \widetilde{\mu}_{j}(\hat{\theta}(t)) \\
\left(\left(\mathscr{A}_{i j}+\Delta A(t)\right) x(t)+\left(\mathscr{B}_{i j}+\Delta B(t)\right) u(t)\right)
\end{aligned}
$$

From equations (21), (13) and (14), the dynamics of the state estimation error is given by

$$
\begin{aligned}
& \dot{e}_{x}(t)=\sum_{i=1}^{r} \sum_{j=1}^{2^{n}} \mu_{i}(\hat{x}(t)) \widetilde{\mu}_{j}(\hat{\theta}(t)) \\
& \left(\left(\mathscr{A}_{i j}-L_{i j} C\right) e_{x}(t)+\Delta A(t) x(t)+\Delta B(t) u(t)\right)
\end{aligned}
$$

Let us now define the parameter estimation error $e_{\theta}(t)$ as

$$
e_{\theta}(t)=\theta(t)-\hat{\theta}(t)
$$

From equation (13), the dynamics of this error is given by

$$
\begin{aligned}
\dot{e}_{\theta}(t)=\sum_{i=1}^{r} \sum_{j=1}^{2^{n}} \mu_{i}(\hat{x}(t)) \widetilde{\mu}_{j}(\hat{\theta}(t)) & \left(\dot{\theta}(t)-K_{i j} C e_{x}(t)+\alpha_{i j} \theta(t)-\alpha_{i j} e_{\theta}(t)\right)
\end{aligned}
$$

Due to the coupling between the errors $e_{\theta}(t)$ and $e_{x}(t)$, it is convenient to consider the augmented vectors $e_{a}(t)$ and $\omega(t)$

$$
e_{a}(t)=\left(\begin{array}{c}
e_{x}(t) \\
e_{\theta}(t)
\end{array}\right), \omega(t)=\left(\begin{array}{c}
x(t) \\
\theta(t) \\
\dot{\theta}(t) \\
u(t)
\end{array}\right)
$$

From (22), (24) and (25), it follows

$$
\dot{e}_{a}(t)=\sum_{i=1}^{r} \sum_{j=1}^{2^{n}} \mu_{i}(\hat{x}(t)) \widetilde{\mu}_{j}(\hat{\theta}(t))\left(\Phi_{i j} e_{a}(t)+\Psi_{i j}(t) \omega(t)\right)
$$

with

$$
\begin{aligned}
\Phi_{i j} & =\left(\begin{array}{ccc}
\mathscr{A}_{i j}-L_{i j} C & 0 \\
-K_{i j} C & -\alpha_{i j}
\end{array}\right) \\
\Psi_{i j}(t) & =\left(\begin{array}{cccc}
\Delta A(t) & 0 & 0 & \Delta B(t) \\
0 & \alpha_{i j} & I & 0
\end{array}\right)
\end{aligned}
$$

Considering (26), our objective is to design joint state and parameter observer with a minimal $\mathscr{L}_{2}$ gain of the transfer from $\omega(t)$ to $e_{a}(t)$. The computation of the observer gains is detailed in the next theorem.

Theorem 1: There exists a joint robust state and parameter observer (13) for a nonlinear time-varying parameter system (3) with an $\mathscr{L}_{2}$ gain from $\omega(t)$ to $e_{a}(t)$ bounded by $\beta(\beta>0)$ if there exists matrices $P_{0}=P_{0}^{T}>0, P_{1}=P_{1}^{T}>0, \Gamma_{2}^{0}, \Gamma_{2}^{1}, \Gamma_{2}^{2}$, $\Gamma_{2}^{3}>0, \bar{\alpha}_{i j}, F_{i j}, R_{i j}$ and scalars $\beta, \lambda_{1}$ and $\lambda_{2}>0$ solution of the optimization problem (28) under LMI constraints (29) and (30)

$$
\min _{P_{0}, P_{1}, R_{i j}, F_{i j}, \bar{\alpha}_{i j}, \lambda_{1}, \lambda_{2}, \Gamma_{2}^{k}} \beta
$$

for $i=1, \ldots, r$ and $j=1,2^{n}$

$$
\Gamma_{2}^{k}<\beta I \text { for } k=0,1,2,3
$$




$$
\left(\begin{array}{cccccccc}
Q_{i j}^{11} & -C^{T} F_{i j}^{T} & 0 & 0 & 0 & 0 & P_{0} \mathscr{A} & P_{0} \mathscr{B} \\
* & -\bar{\alpha}_{i j}-\bar{\alpha}_{i j}^{T}+I_{n} & 0 & \bar{\alpha}_{i j} & P_{1} & 0 & 0 & 0 \\
* & * & -\Gamma_{2}^{0}+\lambda_{1} E_{A}^{T} E_{A} & 0 & 0 & 0 & 0 & 0 \\
* & * & * & -\Gamma_{2}^{1} & 0 & 0 & 0 & 0 \\
* & * & * & * & -\Gamma_{2}^{2} & 0 & 0 & 0 \\
* & * & * & * & * & -\Gamma_{2}^{3}+\lambda_{2} E_{B}^{T} E_{B} & 0 & 0 \\
* & * & * & * & * & * & -\lambda_{1} I & 0 \\
* & * & * & * & * & * & 0 & -\lambda_{2} I
\end{array}\right)<0
$$

with

$$
Q_{i j}^{11}=P_{0} \mathscr{A}_{i j}+\mathscr{A}_{i j}^{T} P_{0}-R_{i j} C-C^{T} R_{i j}^{T}+I_{n_{x}}
$$

The observer gains are given by

$$
\left\{\begin{array}{l}
L_{i j}=P_{0}^{-1} R_{i j} \\
K_{i j}=P_{1}^{-1} F_{i j} \\
\alpha_{i j}=P_{1}^{-1} \bar{\alpha}_{i j}
\end{array}\right.
$$

For the proof of theorem 1, the following lemma is used:

Lemma 1: Consider two matrices $X$ and $Y$ with appropriate dimensions, a time-varying matrice $\Delta(t)$ and a positive scalar $\varepsilon$. The following property is verified

$$
X^{T} \Delta^{T}(t) Y+Y^{T} \Delta(t) X \leq \varepsilon X^{T} X+\varepsilon^{-1} Y^{T} Y
$$

for $\Delta^{T}(t) \Delta(t) \leq I$

Proof: Let us consider the following quadratic Lyapunov function

$$
V\left(e_{a}(t)\right)=e_{a}^{T}(t) P e_{a}(t), P=P^{T}>0
$$

Its time derivative is given by

$$
\begin{aligned}
& \dot{V}(t)=\sum_{i=1}^{r} \sum_{j=1}^{2^{n}} \mu_{i}(\hat{x}(t)) \widetilde{\mu}_{j}(\hat{\theta}(t))\left[e_{a}^{T}(t)\left(\Phi_{i j}^{T} P+P \Phi_{i j}\right) e_{a}(t)\right. \\
& \left.+e_{a}^{T}(t) P \Psi_{i j}(t) \omega(t)+\omega^{T}(t) \Psi_{i j}^{T}(t) P e_{a}(t)\right]
\end{aligned}
$$

It is known that $e_{a}(t)$ asymptotically converges toward zero when $\omega=0$ and that the $\mathscr{L}_{2}$ gain from $\omega$ to $e_{a}$ is bounded by $\beta$ if the following inequality holds

$$
\dot{V}(t)+e_{a}^{T}(t) e_{a}(t)-\omega^{T}(t) \Gamma_{2} \omega(t)<0
$$

with

$$
\Gamma_{2}=\operatorname{diag}\left(\Gamma_{2}^{k}\right), \Gamma_{2}^{k}<\beta I, \text { for } k=0,1,2,3
$$

An adequate choice of $\Gamma_{2}$ allows to attenuate the transfer from some components of $\omega(t)$ to $e_{a}(t)$.

From (35), (36) becomes:

$$
\begin{aligned}
& \sum_{i=1}^{r} \sum_{j=1}^{2^{n}} \mu_{i}(\hat{x}(t)) \mu_{j}(\hat{\theta}(t))\left(\begin{array}{c}
e_{a}(t) \\
\omega(t)
\end{array}\right)^{T} \\
& \left(\left(\begin{array}{cc}
\Phi_{i j}^{T} P+P \Phi_{i j}+I_{n_{x}+n} & P \Psi_{i j}(t) \\
\Psi_{i j}^{T}(t) P & -\Gamma_{2}
\end{array}\right)\right)\left(\begin{array}{c}
e_{a}(t) \\
\omega(t)
\end{array}\right)<0
\end{aligned}
$$

A block diagonal structure for the Lyapunov matrix $P$ is considered:

$$
P=\operatorname{diag}\left(P_{0}, P_{1}\right)
$$

From (23), (27), (37) and (39), (38) holds if

$$
\sum_{i=1}^{r} \sum_{j=1}^{2^{n}} \mu_{i}(\hat{x}(t)) \widetilde{\mu}_{j}(\hat{\theta}(t))\left(Q_{i j}+\mathscr{Q}(t)+\mathscr{Q}^{T}(t)\right)<0
$$

with:

$$
\begin{array}{r}
Q_{i j}=\left(\begin{array}{cccccc}
Q_{i j}^{11} & -C^{T} K_{i j}^{T} P_{1} & 0 & 0 & 0 & 0 \\
* & -P_{1} \alpha_{i j}-\alpha_{i j}^{T} P_{1}+I_{n} & 0 & P_{1} \alpha_{i j} & P_{1} & 0 \\
* & * & -\Gamma_{2}^{0} & 0 & 0 & 0 \\
* & * & * & -\Gamma_{2}^{1} & 0 & 0 \\
* & * & * & * & -\Gamma_{2}^{2} & 0 \\
* & * & * & * & * & -\Gamma_{2}^{3}
\end{array}\right) \\
Q_{i j}^{11}=P_{0} \mathscr{A}_{i j}+\mathscr{A}_{i j}^{T} P_{0}-P_{0} L_{i j} C-C^{T} L_{i j}^{T} P_{0}+I_{n_{x}}
\end{array}
$$

Based on (16) and (17), the time-varying term of (40) can be expressed as:

$$
\begin{aligned}
& \mathscr{Q}(t)=\left(\begin{array}{c}
P_{0} \mathscr{A} \\
0 \\
0 \\
0 \\
0 \\
0
\end{array}\right) \Sigma_{A}(t)\left(\begin{array}{llllll}
0 & 0 & E_{A} & 0 & 0 & 0
\end{array}\right) \\
& +\left(\begin{array}{c}
P_{0} \mathscr{B} \\
0 \\
0 \\
0 \\
0 \\
0
\end{array}\right) \Sigma_{B}(t)\left(\begin{array}{llllll}
0 & 0 & 0 & 0 & 0 & E_{B}
\end{array}\right)
\end{aligned}
$$

Using lemma 1 and property (19), there exists positive scalars $\lambda_{1}$ and $\lambda_{2}$, such that

$$
\mathscr{Q}(t)+\mathscr{Q}^{T}(t)<\left(\begin{array}{cccccc}
\mathscr{Q}^{1} & 0 & 0 & 0 & 0 & 0 \\
0 & 0 & 0 & 0 & 0 & 0 \\
0 & 0 & \lambda_{1} E_{A}^{T} E_{A} & 0 & 0 & 0 \\
0 & 0 & 0 & 0 & 0 & 0 \\
0 & 0 & 0 & 0 & 0 & 0 \\
0 & 0 & 0 & 0 & 0 & \lambda_{2} E_{B}^{T} E_{B}
\end{array}\right)
$$

with:

$$
\mathscr{Q}^{1}=\lambda_{1}^{-1} P_{0} \mathscr{A} \mathscr{A}^{T} P_{0}+\lambda_{2}^{-1} P_{0} \mathscr{B} \mathscr{B}^{T} P_{0}
$$

for $i=1, \ldots, r$ and $j=1, \ldots, 2^{n}$.

From inequality (44), since $\mu_{i}(\hat{x}(t))$ and $\widetilde{\mu}_{j}(\hat{\theta}(t))$ satisfy the convex sum property, with the variable changes (32), the LMI (30) implies (40) and (36). As a consequence, the $\mathscr{L}_{2}$-gain of the transfer from $\omega(t)$ to $e_{a}(t)$ is bounded by $\beta$, which achieves the proof.

\section{NUMERICAL EXAMPLE}

In this section, the proposed approach is applied to a biological wastewater treatment plant. A reduced form of an activated sludge reactor model is considered with only 
the carbon pollution and two state variables.

Starting from the nonlinear equations of the system, a T$\mathrm{S}$ representation is given. Modelling errors (function of a time-varying parameter) are considered. The objective is to synthesize an observer in order to simultaneously estimate the states and the varying parameter.

The process consists in mixing used waters with a rich mixture of bacteria in order to degrade the organic matter [10]. Under specific assumptions, some simplifications can be made and the nonlinear system can be represented with the following equations [7]:

$$
\begin{aligned}
& \dot{x}_{1}(t)=\frac{a(t) x_{1}(t) x_{2}(t)}{x_{2}(t)+b}-x_{1}(t) u(t) \\
& \dot{x}_{2}(t)=-\frac{c a(t) x_{1}(t) x_{2}(t)}{x_{2}(t)+b}+\left(d-x_{2}(t)\right) u(t)
\end{aligned}
$$

with $x_{1}(t)$ and $x_{2}(t)$, the biomass and substrat concentration respectively. The input $u(t)$ represents the dwell-time in the treatment plant. The output is the biomass concentration $\left(y(t)=x_{1}(t)\right)$.

It is assumed that there is a modelling error on the parameter $a$ such that:

$$
a(t)=a+\Delta a(t)
$$

It can also be written as:

$$
a(t)=a+\theta(t) \bar{a}, \quad \theta(t) \in[\underline{\theta}, \bar{\theta}]
$$

with $a=0.5, \bar{a}=0.4$ and $\underline{\theta}=-0.230, \bar{\theta}=0.213$.

Parameters $b, c, d$ have been identified and set to $b=0.07$, $c=0.7$ et $d=2.5$.

From the system nonlinearities, let us consider the following premise variables:

$$
\begin{aligned}
& z_{1}(t)=-u(t) \\
& z_{2}(t)=\frac{(a+\theta(t) \bar{a}) x_{1}(t)}{x_{2}(t)+b}
\end{aligned}
$$

As in the previous sections, using the so-called sector nonlinearity approach, the bounded parameter $\theta(t)$ is described by equations (5) and (6).

Considering (5), the premise variables (49) become:

$$
\begin{aligned}
& z_{1}(t)=-u(t) \\
& z_{2 i}(t)=\frac{\left(a+a_{i}\right) x_{1}(t)}{x_{2}(t)+b}
\end{aligned}
$$

for $i=1,2$ and $a_{1}=\bar{\theta} \bar{a}$ and $a_{2}=\underline{\theta} \bar{a}$.

Then, the following quasi-LPV form is obtained:

$\dot{x}(t)=\sum_{i=1}^{2} \widetilde{\mu}_{i}(\theta(t))\left(\begin{array}{cc}z_{1}(t) & z_{2 i}(t) \\ 0 & -c z_{2 i}(t)+z_{1}(t)\end{array}\right) x(t)+\left(\begin{array}{l}0 \\ d\end{array}\right) u(t)$

For the considered simulation, the maximum and minimum values of the premisse variables have been computed: $z_{1}(t) \in[-1,-0.2], \quad z_{21}(t) \in[0.0047,4.3894]$ and $z_{22}(t) \in$ $[0.0033,3.0587]$. That knowledge allows to deduce a T-S form. Indeed, using the convex polytopic transformation, two partitions for each premise variables are constructed as follows:

$$
\left\{\begin{array}{l}
z_{1}(t)=F_{11}\left(z_{1}\right) \bar{z}_{1}+F_{12}\left(z_{1}\right) \underline{z}_{1} \\
z_{2 i}(t)=F_{2 i}^{1}\left(z_{2 i}\right) \bar{z}_{2 i}+F_{2 i}^{2}\left(z_{2 i}\right) \underline{z}_{2 i}, \text { for } i=1,2
\end{array}\right.
$$

with

$$
\begin{aligned}
& F_{11}\left(z_{1}\right)=\frac{z_{1}(t)-\underline{z}_{1}}{\bar{z}_{1}-\underline{z}_{1}}, F_{12}\left(z_{1}\right)=\frac{\bar{z}_{1}-z_{1}(t)}{\bar{z}_{1}-\underline{z}_{1}} \\
& F_{2 i}^{1}\left(z_{2 i}\right)=\frac{z_{2 i}(t)-\underline{z}_{2 i}}{\bar{z}_{2 i}-\underline{z}_{2 i}}, F_{2 i}^{2}\left(z_{2 i}\right)=\frac{\bar{z}_{2 i}-z_{2 i}(t)}{\bar{z}_{2 i}-\underline{z}_{2 i}}
\end{aligned}
$$

where the scalars $\bar{z}_{1}, \underline{z}_{1}, \bar{z}_{2 i}$ and $\underline{z}_{2 i}$ are defined as

$$
\begin{aligned}
& \bar{z}_{1}=\max _{u} z_{1}(t), \underline{z}_{1}=\min _{u} z_{1}(t) \\
& \bar{z}_{2 i}=\max _{x} z_{2 i}(t), \underline{z}_{2 i}=\min _{x} z_{2 i}(t)
\end{aligned}
$$

The submodels are defined by the pairs $\left(\mathscr{A}_{i j}, \mathscr{B}_{i j}\right)$ with $i=1, \ldots, 4$ and $j=1,2$. Due to the choice of premise variables, all the $\mathscr{B}_{i j}$ matrices are equal to $B^{T}=\left[\begin{array}{ll}0 & d\end{array}\right]$. The matrices $\mathscr{A}_{i j}$ are given by:

$$
\begin{aligned}
& \mathscr{A}_{11}=\left(\begin{array}{cc}
\bar{z}_{1} & \bar{z}_{21} \\
0 & -c \bar{z}_{21}+\bar{z}_{1}
\end{array}\right), \mathscr{A}_{12}=\left(\begin{array}{cc}
\bar{z}_{1} & \bar{z}_{22} \\
0 & -c \bar{z}_{22}+\bar{z}_{1}
\end{array}\right) \\
& \mathscr{A}_{21}=\left(\begin{array}{cc}
\bar{z}_{1} & \underline{z}_{21} \\
0 & -c \underline{z}_{21}+\bar{z}_{1}
\end{array}\right), \mathscr{A}_{22}=\left(\begin{array}{cc}
\bar{z}_{1} & \underline{z}_{22} \\
0 & -c \underline{z}_{22}+\bar{z}_{1}
\end{array}\right) \\
& \mathscr{A}_{31}=\left(\begin{array}{cc}
\underline{z}_{1} & \bar{z}_{21} \\
0 & -c \bar{z}_{21}+\underline{z}_{1}
\end{array}\right), \mathscr{A}_{32}=\left(\begin{array}{cc}
\underline{z}_{1} & \bar{z}_{22} \\
0 & -c \bar{z}_{22}+\underline{z}_{1}
\end{array}\right) \\
& \mathscr{A}_{41}=\left(\begin{array}{cc}
\underline{z}_{1} & \underline{z}_{21} \\
0 & -c \underline{z}_{21}+\underline{z}_{1}
\end{array}\right), \mathscr{A}_{42}=\left(\begin{array}{cc}
\underline{z}_{1} & \underline{z}_{22} \\
0 & -c \underline{z}_{22}+\underline{z}_{1}
\end{array}\right)
\end{aligned}
$$

The T-S model is obtained by an interpolation of the eight previous submodels:

$$
\dot{x}(t)=\sum_{i=1}^{4} \sum_{j=1}^{2} \mu_{i j}(z(t)) \tilde{\mu}_{j}(\theta(t))\left(\mathscr{A}_{i j} x(t)+B u(t)\right)
$$

The weighting functions $\tilde{\mu}_{j}(\theta(t))$ are calculated from equations (6) and $\mu_{i j}(z(t))$ as the following:

$$
\mu_{i j}(z(t))=F_{1}^{\sigma_{i}^{l}}\left(z_{1}(t)\right) F_{2 j}^{\sigma_{i}^{l}}\left(z_{2 j}(t)\right)
$$

where $\sigma_{i}^{l}$ represents the $l^{\text {th }}$ partition. For example, we have:

$$
\begin{aligned}
& \mu_{11}(z(t))=F_{11}\left(z_{1}(t)\right) F_{21}^{1}\left(z_{21}(t)\right) \\
& \mu_{12}(z(t))=F_{11}\left(z_{1}(t)\right) F_{22}^{1}\left(z_{22}(t)\right)
\end{aligned}
$$

Then, from the nonlinear system (46), an equivalent T-S model is obtained and given by (55). As mentioned at the begining of this section, the objective is to synthesize a robust state and parameter observer applying the proposed approach. To illustrate the time-varying parameter effect on the system, figure 1 depicts the states in the nominal case (with $a(t)=a$ ) and in the time-varying case $(a(t)=$ $a+\theta(t) \bar{a})$.

The system input, the state variables and their estimates, the time-varying parameter and its estimate are depicted in the figures 2,3 and 4 respectively. The initial conditions are $x_{0}=\left(\begin{array}{ll}0.1 & 1.5\end{array}\right)$ for the system state and $\hat{x}_{a}(0)=$ $\left(\begin{array}{lll}0.09 & 2.3 & 0\end{array}\right)$ for the joint state and parameter estimate.

From the depicted figures, one can conclude on the robustness of the synthetized state observer, since the two states are perfectly estimated as well as the time-varying parameter $\theta(t)$. 

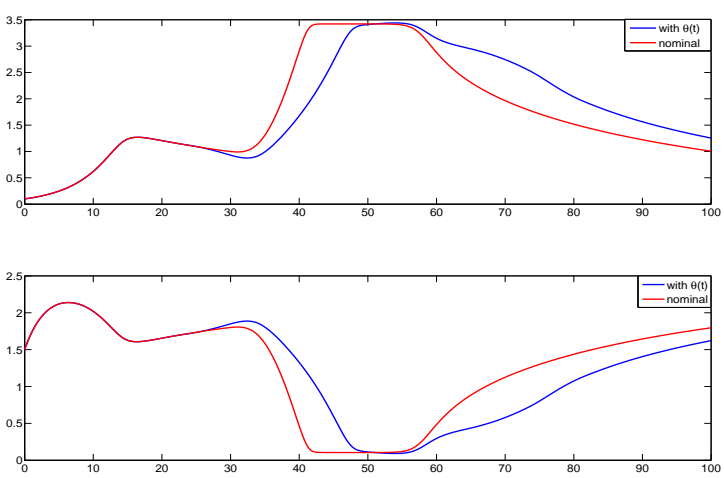

Fig. 1. System states with and without $\theta(t)$

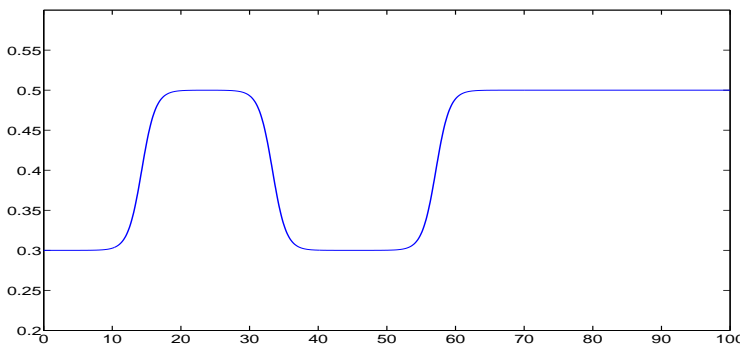

Fig. 2. System input

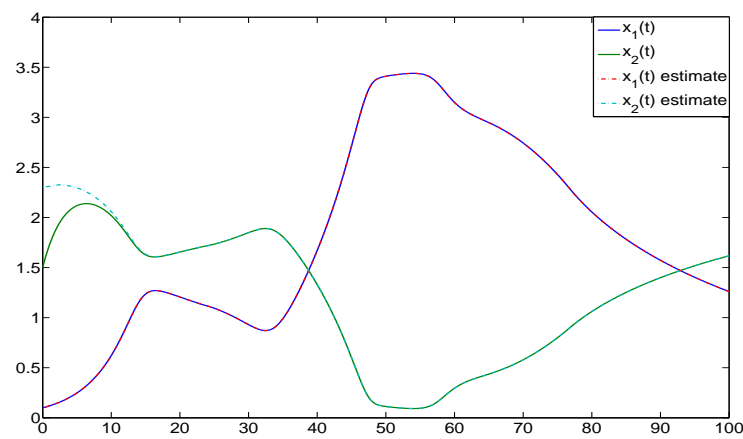

Fig. 3. System states and their estimates

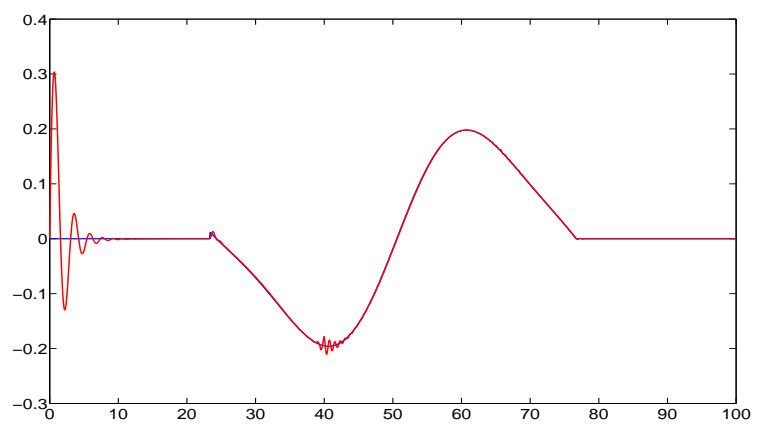

Fig. 4. Time-varying parameter and its estimate

\section{CONCLUSion}

In the present paper, a new systematic procedure is presented to deal with the state and parameter estimation for nonlinear time-varying systems. It consists in transforming the original system into a Takagi-Sugeno model, based on the sector nonlinearity approach and the convex polytopic transformation. This transformation has the major interest to exactly represent the system without any loss of informations. Then a state and parameter observer can be designed by minimizing the $\mathscr{L}_{2}$ gain from the augmented input to the estimation errors. The chosen application example is an activated sludge reactor with modeling uncertainties represented by an unknown time-varying parameter. From the nonlinear equations of the system, a T-S model is derived. The proposed observer is synthetized and the obtained results illustrate its performance.

\section{REFERENCES}

[1] A. Akhenak, M. Chadli, J. Ragot, and D. Maquin. Design of sliding mode unknown input observer for uncertain Takagi-Sugeno model. In $15^{\text {th }}$ Mediterranean Conference on Control and Automation, MED07, Athens, Greece, 2007.

[2] M. Basseville. On-board component fault detection and isolation using the statistical local approach. Automatica, 34(11):1391-1415, 1998.

[3] G. Besançon. Remarks on nonlinear adaptive observer design. Systems and Control, 41(4):271-280, 2000.

[4] S. Bezzaoucha, B. Marx, D. Maquin, and J. Ragot. Linear feedback control input under actuator saturation : A Takagi-Sugeno approach. In $2^{\text {nd }}$ International Conference on Systems and Control (ICSC'12), Marrakech, Morocco, June 20-22 2012.

[5] J. Chen and R.J. Patton. Robust Model Based Fault Diagnosis for Dynamic Systems. Kluwer Academic Publishers, Boston, Dordrecht, London, 1999.

[6] Y. Cho and R. Rajamani. A systematic approach to adaptive synthesis for nonlinear systems. IEEE Transactions on Automatic Control, 42(4):534-537, 1997.

[7] D. Ichalal, B. Marx, D. Maquin, and J. Ragot. Estimation d'état des systèmes non linéaires incertains sous forme multimodèle de type Takagi-Sugeno. In $1^{\text {st }}$ Colloque International Francophone, Ingénierie et Environnement CIFIE, Anaba, Algérie, 2010 (in french).

[8] B. Marx, D. Koenig, and J. Ragot. Design of observers for TakagiSugeno descriptor systems with unknown inputs and application to fault diagnosis. IET Control Theory and Applications, 1(5):1487$1495,2007$.

[9] A.M. Nagy, G. Mourot, B. Marx, J. Ragot, and G. Schutz. Systematic multimodeling methodology applied to an activated sludge reactor model. Industrial Engineering Chemical Research, 49(6):2790-2799, 2010.

[10] A. Nagy Kiss, B. Marx, G. Mourot, G. Schultz, and J. Ragot. State estimation of two time-scale multiple models. Application to wastewater treatment plant. Control Engineering Practice, 19(11):1354-1362, 2011.

[11] R. Rajamani and J.K. Hedrich. Adaptive observer for active automotive suspensions: theory and experiment. IEEE Transactions on Control Systems Technology, 3(1):86-93, 1995.

[12] T. Takagi and M. Sugeno. Fuzzy identification of systems and its applications to modeling and control. IEEE Transactions on Systems, Man, and Cybernetics, 15(1):116-132, 1985.

[13] K. Tanaka and H.O. Wang. Fuzzy Control Systems Design and Analysis: A Linear Matrix Inequality Approach. John Wiley \& Sons, Inc., 2001.

[14] Q. Zhang and A. Xu. Implicit adaptive observers for a class of nonlinear systems. In American Control Conference, ACC, Arlington, Virginia, 2001. 